\title{
Improving patient discharge process using electronic medication input tool and on-line guide to arranging follow-ups
}

\author{
Rory Barr, Kuen Yeow Chin, Keefai Yeong
}

\begin{abstract}
Complete, accurate and timely discharge summaries (TTOs) enable effective communication between hospital teams and GPs. It can prevent adverse events and reduce hospital readmission rates (1). If the discharge summary does not contain important information (e.g. follow-up arrangements, accurate discharge medication list), or if follow-up arrangements are not made, then patient care and outcome can be adversely affected $(2,3)$.

An electronic Medication Input Wizard was developed to improve the quality and reduce the error rates of TTOs. The Wizard makes entering drug information faster; prompts for reasons medication changes; provides examples for Controlled Drug (CD) prescribing; and prompts to refer patients taking warfarin to anticoagulation clinic. An on-line guide was developed which explains how to arrange investigations and appointments. Retrospective studies of TTOs were carried out before and after these interventions, analysing documentation of medication and completion of intended follow-up arrangements.
\end{abstract}

A baseline audit found $65 \%$ of medication changes on TTOs were not clearly documented, and only $8 \%$ with changes documented reasons. $40 \%$ of prescriptions for CDs were incorrect delaying discharge by 4.9 hours per patient. $80 \%$ of intended follow-ups actually happened.

After intervention, TTOs written using the Wizard had $100 \%$ of medication changes documented, and $75 \%$ were documented with reasons. CD errors decreased to $28 \%$ ( $76 \%$ of errors were done without using the Wizard). Follow-up arrangements that occurred increased to $86 \%$. A survey showed $78 \%$ of Doctors reported the Wizard was faster than typing the details in separate textboxes, and $94 \%$ believed it was beneficial.

Systems should be optimised to encourage better documentation of medication details and reduce prescribing errors. Guides that explain how to make follow-up arrangements should be accessible to Doctors, to make sure follow-ups are organised correctly.

\section{Problem}

Patients discharged from hospital receive a discharge summary (TTO), a copy of which is also posted to the patient's GP. In our hospital, TTOs are written electronically and include details relating to the admission; diagnosis, tests and treatment, medication details, and follow up arrangements. The doctor preparing the discharge summary is also responsible for detailing and arranging any outpatient follow-up investigations or clinic appointments as needed.

However, several issues can adversely affect the patient discharge process.

(i) Medication Input: using the current system, the doctor has three different text boxes relating to medication to type in - Medication on Admission, Medication Changes, and Medication on Discharge. This unnecessary repetition (the same medication might need to be typed in each of the three boxes) increases the risk of errors. Some doctors do not fill in the Medication on Admission or Medication Changes sections (perhaps due to time pressure), therefore changes to medications are not documented and explained to pharmacists, the GP or patients. This often results in a Pharmacist having to contact the Doctor to ask for more information. (ii) Prescribing errors for Controlled Drugs (CDs): CDs need to be prescribed in accordance with legal guidelines. Incorrect prescriptions on Discharge Summaries mean a pharmacist has to contact the Doctor to rewrite the Discharge Summary, and send the corrected copy down to pharmacy, wasting time for Pharmacists, Doctors, and ultimately delaying discharge for patients.

(iii) Follow up arrangements: Patients discharged on Warfarin should have a follow up appointment made to monitor INR levels. If this is not done, the patient is at risk of having a sub-therapeutic INR and embolic event, or too high an INR, and having a bleed. If other intended outpatient follow-up arrangements for clinics or investigations are not made then this could lead to adverse outcomes for patients.

\section{Background}

Complete, accurate, and timely discharge summaries enable effective communication between hospital teams and GPs, prevent adverse events, and reduce readmission rates to hospital (1). If the discharge summary does not contain certain items (follow up arrangements and pending test results, and an accurate discharge medication list) then patient care and health outcomes can be adversely affected $(2,3)$. 
Discharge medication lists, medication changes, and the reason for medication changes (if documented) have been found to be inaccurate in $35.7 \%, 29.5 \%$, and $37.7 \%$ of discharge summaries respectively 4 . Adverse drug events after hospital discharge increases risk of morbidity and mortality 5 , so it is clear that improving accuracy in recording medications on discharge can contribute to patient safety. Poor communication of medical information at transition points has been shown to be responsible for up to $50 \%$ of all medication errors, and $20 \%$ of adverse drug events in hospital6. Although medication errors are common, not all are harmful - studies have shown that less than $1 \%$ of all medication errors result in an adverse drug event, while up to $6.7 \%$ create potential adverse drug events, when examined by chart reviews $(7,8,9)$.

Communication of follow-up plans for further investigation is often inadequate, with $27.7 \%$ of summaries rated as insufficient (10). Studies have shown documented follow-up plans on discharge summaries are absent between $2 \%-43 \%$ of cases (11).

\section{Baseline Measurement}

Medication errors on discharge summaries are very common; an audit in St Peter's pharmacy in 2011 found an average of 2.04 medication errors per discharge summary. A retrospective study of all electronic TTOs from Jan 1st 2012 to Dec 31st 2012 showed that $9 \%(3,258$ TTOs $)$ contained at least one of 20 of the most commonly prescribed CDs. In March 2013, 40\% (19/48) of discharge summaries processed by the hospital pharmacy with a CD were prescribed incorrectly, resulting in an average delay of 4.9 hours per patient (range 3 hours 15 minutes to 7 hours 15 minutes). This suggests 1,303 patients a year could have a CD prescribed incorrectly on discharge, either resulting in an average delay of 4.9 hours to discharge if medication provided by hospital pharmacy, or if prescriptions are written incorrectly on an FP10 form to be processed by a community pharmacist, then such patients would not be able to get that medication at all due to the error.

An audit in October 2011 investigated the proportion of outpatient follow-up clinics/investigations written on TTOs that actually happened13. 20 patients were selected from a busy respiratory and general medical ward. Notes were examined for details of follow up, this was compared with the discharge summary and the interventions (appointments \& investigations) subsequently arranged. A follow-up prompt sheet was then placed in patient notes. Results showed $79 \%$ of intended interventions happened. A retrospective study of all electronic TTOs produced in 2012 showed that $7 \%$ of patients were discharged on Warfarin (2,517 p.a.). TTOs for all patients discharged on Warfarin for 7 days in March 2013 checked with the anticoagulation service two weeks later to see how many patients were referred. $40 \%$ (17/43) had not been referred for anticoagulation clinic follow-up to monitor their INR .

A retrospective study of medication documentation on TTOs was carried out on all TTOs written on May 1st 2013. The proportion of discharge summaries including details of Medication on Admission, Medication Changes, and Medication on Discharge was measured, as well as assessing if all medication changes and if reasons for changes were documented. Each discharge summary was scored as follows $-0=$ no medication change, $1=$ medication change(s) not documented, 2=medication(s) changed, documented, no reasons given, $3=$ medication(s) changed, documented, and reasons given, $\mathrm{n} / \mathrm{a}=$ cannot tell if any medications changed. A total of 92 TTOs were written - 53\% (49/92) had clear changes (medication on admission was not the same as medication on discharge), and 34\% (31/92) were not clear enough to ascertain if there had been any medication changes (only medication on admission or medication on discharge was listed). Of the TTOs with identifiable medication changes, 65\% (32/49) were not documented in "medication changes", $27 \%$ (13/49) documented all changes, and 8\% (4/49) documented and provided reasons for all changes.

See supplementary file: ds2016.xls - "baselineData"

\section{Design}

The key cause of problems in making follow up arrangements correctly is a lack of knowledge about how to make those arrangements, coupled with pressure on Doctors to discharge patients as quickly as possible. The key cause for prescription errors and incomplete information relating to medication changes on discharge summaries is due to systemic inefficiency meaning that filling in all required information takes an unnecessary length of time, so some Doctors chose to not document medication on admission and mediation changes to speed up the process of discharging patients.

An intervention to improve the process and quality of discharge summaries must therefore directly address these issues, and help increase knowledge and awareness so that follow up arrangements are made correctly. An on-line guide which can be quickly accessed from a link directly adjacent to the "Follow Up Arrangements" box on a discharge summary system was developed to provide succinct, useful information to tell doctors how to arrange clinics and investigations for patients being discharged.

To speed up the process of entering medications and encourage more thorough documentation about medication on discharge and medication changes, an electronic Medication Input Wizard was developed. As well as speeding up the entry of medication information, this wizard prompts doctors if they are prescribing a CD and asks if they have prescribed it correctly (while showing examples of how to prescribe CDs properly). The wizard will also prompt Doctors discharging patients on warfarin to make sure that the patient is referred to the hospital's anti-coagulation clinic, with details about how to do so.

\section{Strategy}

PDSA Cycle 1 - ask another Doctor to use Medication Input Wizard, and provide feedback.

Outcome - suggested changes to hide input textboxes when not needed. 
PDSA Cycle 2 - ask Junior Doctor and Divisional Director of Medicine to use Medication Input Wizard, and provide feedback

Outcome - Divisional Director suggested adding "Reason for change" textbox, to encourage better explanation of why medications started, stopped, increased or decreased.

PDSA Cycle 3 - Doctors to test Medication Input Wizard, and FollowUp Arrangements guide.

Outcome - bug discovered relating to javascript version running on Internet Explorer 6. Suggested additions to Follow-Up guide.

PDSA Cycle 4 - Bug fixed, Follow-Up Arrangements guide amended. Retest by Doctors.

Outcome - no bugs or further changes as a result. Ready for full end-to-end testing.

PDSA Cycle 5 - Full regression end-to-end testing.

Outcome - no bugs, go Live with Medication Input Wizard, and Follow-Up Arrangements guide. Communicate changes by email, posters, and presentations at Teaching and Grand Rounds.

See supplementary file: ds2015.docx - "PDSA cycles"

\section{Post-Measurement}

The Medication Input Wizard (Wizard) and Follow-Up Arrangements webpages went Live in May 2013. Training, posters, and presentations were given to explain their purposes and demonstrate their use. To measure the impact of the Wizard, all TTOs from the 5th of June 2013 were analysed and scored using the same system as before. Use of the Wizard was not mandatory, so users needed to click on a hyperlink to open the tool. The Wizard was also only available in templates for Inpatients, so it was not available for Day Surgery patients, or A\&E attendances. Of the 124 TTOs produced on 5th of June, 16 had no medication. The only way to confirm if a TTO was written using the wizard is by seeing if Medication Changes have been documented in the format automatically used by the Wizard. If there have been no changes to medication, it is possible that the Wizard was used, but not possible to tell after it has been written. Of the 108 TTOs with medication, $7 \%$ (8) were written using the Wizard (though as described above the real number could be higher). Given that the Wizard is most useful when there are changes to medications, it is more informative to use that comparison - of the 45 TTOs with medication changes, $18 \%(8 / 45)$ were created using the Wizard.

Documentation of medication changes improved significantly by using the wizard - at baseline only $35 \%$ of medication changes were documented (17/49), but TTOs written using the Wizard documented $100 \%$ of changes (8/8). The documentation of reasons for medication changes also improved, from baseline of $8 \%(4 / 49)$ to $75 \%(6 / 8)$. Using the Wizard results in much better documentation of medication changes, providing more information to patients, relatives, pharmacists and GPs.
A survey was conducted among Doctors in the Trust, in July 2013. $78 \%$ of respondents (14/18) reported using the Wizard; $72 \%(13 / 18)$ reported it was Slightly Faster or Much Faster to enter medication details. $61 \%(11 / 18)$ reported documenting more medication changes than before as a result of the Wizard. 83\% (15/18) reported that the warning messages and examples when prescribing CDs and warfarin were beneficial. $94 \%$ of respondents (17/18) believed the Wizard was beneficial.

With regard to the guide to follow-up arrangements, only $28 \%(5 / 18)$ reported using this themselves, but 50\% (9/18) believed the guide would be beneficial ( $39 \%, 7 / 18$ felt unable to say as they hadn't used it, and $11 \%, 2 / 18$ did not believe it would be beneficial).

An audit was carried out of anticoagulation clinic referrals for patients discharged on warfarin between July 1st 2013 and July 7th 2013 . Only $43 \%$ of patients $(13 / 30)$ were referred to the anticoagulation clinic. This is a decrease from baseline rate of $60 \%$ $(23 / 40)$. Including a prompt in the optional Wizard when a patient is discharged on warfarin has not increased the referral rate.

An audit was carried out to see what proportion of outpatient clinics and investigations listed on TTOs happened after discharge. 108 TTOs of all patients discharged between 1st June and 6th June were analysed. 42 patients were found to have clinics, investigations or other follow ups listed under "Follow Up Arrangements" on their TTO. The 42 patients had a total of 59 separate follow up items, of which $86 \%$ actually occurred (51/59). The follow ups that did not happen were: a request for the GP to book two separate hospital outpatient clinic appointments; a repeat abdominal UltraSound scan; a 24-hour cardiac monitor and two clinic appointments; a Stroke Clinic follow up appointment; and a Community Dietitian follow up. This audit shows an improvement from the baseline rate of $80 \%$, suggesting that the intervention of the follow-up guide has helped.

An audit was carried out assessing the prescription of CDs on TTOs reviewed by pharmacy. $28 \%$ (17/60) had errors. Of the errors, $76 \%$ of those TTOs were written without using the Medication Input Wizard (13/17). The implementation of the Wizard has led to a decrease in Controlled Drug prescribing errors, from $40 \%$ to $28 \%$. It is significant that $76 \%$ of the errors were written without using the Wizard.

See supplementary file: ds2017.xls - "compData"

\section{Lessons and Limitations}

This project involved several changes, each implemented with varying degrees of success. With regard to the Medication Input Wizard, it clearly achieved its objectives when it was used however, as its use was optional in the Trust, and not available in all discharge summaries (e.g. for Day Surgery or A\&E patients) not all staff used it. If the aim is to ensure that all medication changes are clearly documented, then making use of this tool mandatory would be one way to achieve that. It would also lead to more medication changes having reasons documented. 
The Wizard included warnings about prescribing CDs, which helped reduce the number of errors from $40 \%$ of CD prescribed incorrectly on TTOs to $28 \%$. Since $76 \%$ of those errors were done without using the Wizard, it is another reason to consider making its use mandatory. The Wizard also provided a reminder to refer patients prescribed warfarin on discharge to the anticoagulation clinic. Before our intervention, $60 \%$ (23/40) of patients were correctly referred to the anticoagulation clinic. after the change, that actually reduced to $43 \%(13 / 30)$.

The reasons for this low referral rate are multifaceted.

-The local process for referral to the anticoagulation clinic is quite a time consuming one, requiring a 3 page form to be manually filled in by the Doctor, and sent to the Clinic which is only open Monday to Friday from $9 \mathrm{am}-5 \mathrm{pm}$.

-Some Doctors might judge that the current admission will not affect the patient's warfarin status, and they should be followed up as usual, not requiring a referral.

- The Wizard was not used for all TTOs, so the warning would not have been seen in all these cases. Moreover, even if the Doctor was prompted to refer a patient for anticoagulation follow up, this is no guarantee that they will.

Increasing rates of referrals for anticoagulation follow up is a complex area, and I would suggest requires a streamlining of the process for referrals, with an automatic electronic referral generated when a patient is discharged on warfarin.

\section{Conclusion}

The Medication Input Wizard has proved its effectiveness - 94\% of Doctors surveyed thought it was beneficial, $72 \%$ agreed it made inputting medication details faster, and 100\% TTOs written using the Wizard documented all changes clearly, with $75 \%$ including the reasons for such changes. That compares to only $35 \%$ of TTOs (with medication changes) clearly documenting those changes, and just $8 \%$ documenting changes and the reasons when not using the Wizard. The Medication Wizard has also reduced errors in prescribing Controlled Drugs, from $40 \%$ to $28 \%$. Most of the errors $(76 \%)$ were written without using the Wizard. In summary, using the Wizard is faster for Doctors, improves communication between hospital doctors, pharmacists, and GPs, reduces errors prescribing CDs, preventing unnecessary delays in discharge and associated costs, and improves patient safety by providing clearer reasons for medication changes.

Given the benefits of the Wizard, it is worth considering making use of the tool mandatory when writing TTOs, or making it the default entry mechanism instead of requiring a user to click on a link and open a pop-up window.

Providing an easily accessible guide on how to arrange outpatient clinics and investigations is useful, particularly to doctors who are new to a Trust, however that in itself is no guarantee that more intended follow-ups will be arranged properly. As with anticoagulation clinic referrals, a reminder might not be enough to overcome a time-consuming process. I suggest referral processes themselves need to be made as efficient as possible, with referrals automatically generated from discharge summaries (so they already include most of the required information), and sent on-line rather than requiring a Doctor to print them out, and fax or physically post the referral.

\section{References}

1 Walraven C, Seth R; Effect of Discharge Summary Availability durin Post-discharge Visits on Hospital Readmission. J Gen Intern Med 2002, 17:186-19

2 Moore C, McGinn T, Halm E: Tying up loose ends: discharging patients with unresolved medical issues. Arch Intern Med 2007, 167:1305-1311.

3 Perren A, Previsdomini M, Cerutti B, Soldini D, Donghi D, Marone $\mathrm{C}$ : Omitted and unjustified medications in the discharge summary. Qual Saf Health Care 2009, 18:205-208

4 Legault et al. Quality of discharge summaries prepared by first year internal medicine residents. BMC Medical Education 2012, 12:77, http://www.biomedcentral.com/1472-6920/12/77

5 Forster AJ, Murff HJ, Peterson JF, Gandhi TK, Bates DW: The incidence and severity of adverse events affecting patients after discharge from the hospital. Ann Intern Med 2003, 138:161-167

6 Institute for Healthcare Improvement (2011). Medication reconciliation review. http://www.ihi.org/knowledge/Pages/Tools/MedicationReconciliation

Review.aspx Accessed 14th July 2013

7 Bates DW, Cullen FJ, Naird N et al. Incidence of adverse drug events and potential adverse drug events. Implications for prevention. ADE Prevention Study Group. J Am Med Assoc 1995; 274:29-34

8 Bates DW, Boyle DL, Vander Vliet MB, Schneider J, Leape L. Relationship between medication errors and adverse drug events. J Gen Intern Med 1995; 10: 199-205

9 Kaushal R, Bates DW, Landrigan C, McKenna KJ, Clapp MD, Federico $\mathrm{F}$ et al. Medication errors and adverse drug events in paediatric inpatients. J Am Med Assoc 2001; 285:2114-2120

10 Legault et al. Quality of discharge summaries prepared by first year internal medicine residents. BMC Medical Education 2012, 12:77, http://www.biomedcentral.com/1472-6920/12/77

11 Krilaani et al. Deficits in Communication and Information Transfer between Hospital-Based and Primary Care Physicians. JAMA, 2007;297:831-841

\section{Declaration of interests}


After designing and implementing this project in May 2013, Dr Rory Barr became Director of CB Healthcare Solutions, which offers Consultancy advice to Healthcare bodies, and seeks to license the Medication Input Wizard described in this paper. Kuen Yeow Chin is also a director of CB Healthcare Solutions.

\section{Acknowledgements}

The techological changes implemented in the Trust would not have been possible without the cooperation and support of IT Lead Andrew Davis.

This project received the backing of the Trust's Chief Executive Andrew Liles, and Director of Finance and Operations Simon Marshall.

Pharmacist Katie Reygate advised on issues relating to Controlled Drugs, and helped complete audits of medication errors. 